High Utility Dataset with transaction database uses datasets to support dataset is not enough to reflect the original utility dataset. It reflects with unit profit list of whole transaction list to get summarized data list values to obtain the profit.

Frequent Pattern Mining: FPM [4] in a shopping database refers to the blocks of products referred that are frequently purchased by the customers and are applied to various application to domains, such as market strategy, financial forecast, bioinformatics, and mobile environments and also to different kinds of databases such as transactional databases, tracking databases and interval of times databases in current fundamental researches.

Apriori Algorithm: It is an "Association Mining Rule between Blocks of Data in Large Databases". Association Rule Mining is not only applied to market basket data. The main challenge in association rule is to identify frequent datasets. Finding frequent Itemset is important measure in ARM. The trouble solution is to be straight forward and focus on how to generate frequent datasets.

In this Paper is organized as follows: Chapter I explains about High Utility Mining Introduction, Chapter II explains about Literature survey of the project, In Chapter III contains objective related to project. In Chapter IV contains System Architecture, diagrams, figures which are necessary for the implementation of the proposed system, Chapter V contains proposed system, Chapter VI contains Implementation results and chapter VII concludes the discussion.

\section{LITERATURE SURVEY}

\section{1 "Efficient Algorithms for Mining Top-K High Utility Dataset"}

Frequent Itemset Mining discovers a higher amount of frequent data is used with lower-value dataset. It missed with lots of information on datasets having lower selling price. High Utility datasets mining, to find all datasets having a profit meeting a client characterized least utility. Setting minimum utility is trouble for the client, so finding a lowest utility end point by experiment for the clients.

The searching of related products details to space for HUD mining is somewhat difficult to the clients because user setting of a lower utility dataset can be high utility is the drawback in the system, so that the proposed algorithm have Top $\mathrm{K}$ values to attain related products and data with desired number of compared items. Setting of threshold value to the product data by user are problem to overcome this, effective algorithm are used they are TKU and TKO. Without the need of specifying the minimum threshold value Top K algorithms are used effectively. TKU algorithm for mining Top-k high utility dataset uses techniques to raise the searching related product space items and border minimum utility profit effectively. The transaction weighted model facilitates performance of the system mining activity is the proposed system of mining utility dataset enhancement.

\section{2 "High Utility Dataset Mining from Transaction Database Using Up-Growth and Up-Growth+ Algorithm"}

The mining performance deduces effectiveness in terms of executing utilization and power space. The utility pattern tree views the original database to operate in a data structured way. The information's are maintained in a small tree-like data structure in high utility dataset. UP-Tree for recording the information datasets and the information with high utilities have four effective strategies to reduce the related search product area in an database and quantity of users in the system with Discarding Unpromising Items and Nodes.

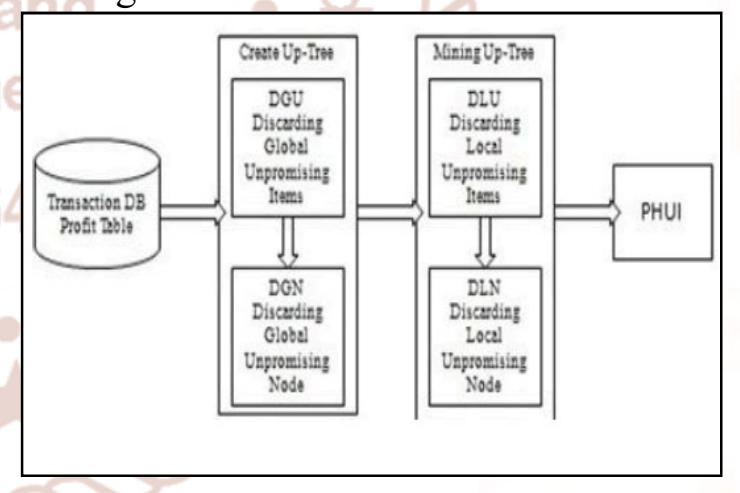

Figure 1 represents Four strategies used in potential HUI

It Advantages on scanning DB twice, when database is updated it reduces unwanted calculation, easy to implementation, less power space and execution duration are required. The Proposed algorithms have effective UP Growth with improved less memory consumption of system and outer perform the system to potential high utility processing time.

\section{3 "Mining High Utility Patterns in One Phase without Generating Candidates"}

Apriori calculations works on this situation with solution to obtain two-forms, they are user generation 
tactics with one condition that is incapable and not scalable with large databases. It suffers from scalability issue due to the more quantity of applicants. To discover high utility pattern in a word without generating applicants in the algorithm. It is affiliated to frequent pattern mining, includes repression part of mining. FPM Algorithms for mining high utility patterns into three subdivisions, they are distance search, height search, and cross search. Utility Mining measures are categorized as experimental measure, patented measure, and phrase measure. In One Part Mining without applicant Generation namely Dead Detection of High Utility Patterns, this degrades number of designs to be detailed.

HUP growth performs design detailed tactics for searching utility higher bounding. The dead Detection of High Utility Pattern shares framework which discovers high utility design without applicant generation. Benefaction contains a linear data structure with applicant generation tactics take up by Apriori algorithm and their data structure not observe the real profit data.

\section{4 "A Review on Infrequent Weighted Itemset Mining Using Frequent Pattern Growth"}

IPM is a dataset mining in frequency occurrence which follows the rules dataset is lower than or equality to lower profit. The mining technique on infrequent weighted dataset uses algorithms of Apriori and frequent pattern growth. Mining infrequent patterns that are focused on mining negative patterns and support for expectation based on ranked series and indirect affiliations.

Mining weighted frequent patterns of mining techniques are developed for dataset mining algorithm used to push weighted values and provide a tree structure of traversal bottomup technique. In mining, frequent pattern does not have different weight point of the data. The frequent datasets are patterns or data or like datasets, substructures, or subsequences of the sets list that come out in a dataset frequently.

Weighted frequencies have tree representation to structures that are like weighted point values on the branch to arrange with frequent buyers order and about its transactions. Infrequent datasets are consider with all datasets that are not extracted by standard frequent dataset generations calculations such as Apriori calculations and frequent pattern growth. The problem statement with mining of infrequent weighted datasets provides with minimum execution time and minimum storage is implemented for the technique.

\section{5 "Mining High Utility Datasets - A Recent Survey"}

Association rule mining plays a vital role in data mining. It aims at searching for interesting pattern among items in a dense data set or database and discovers association rules among the large number of datasets. The importance of ARM is increasing with the demand of finding frequent patterns from large data resources. To discover new relations in Affiliation Rule Mining to different datasets in the databases. Mining dataset Utility is an extension of frequent dataset mining, which discovers datasets that are occurs frequently. The fundamental principle of Frequent Dataset Mining is to identify all the frequent datasets in a database. The initial solution of frequent pattern mining, candidate set generation-and-test paradigm of Apriori Algorithm has many disadvantage that includes multiple database views and generates many user datasets. High Utility Dataset Mining Approach follows

\section{Mining with Expected High Utility}

UMining for High utility upper bound

Isolated Dataset Discarding Calculation

Facts of High Utility Mining Algorithm

$>$ Display and Two series Algorithm Utility Pattern and Growth+ Algorithm

Mining high utility datasets depends on factors like reducing the related product search, quantity of scans on original database, and improving performance. High Utility Datasets are mostly used in real life applications.

\section{OBJECTIVE}

The fundamental objective is to show Utility Mining in the datasets with highest utilities, by considering profit, volume, expenditure or other user favorites. To improve the system performance, effective rating with evaluation of extensive experiments with encrypted data which is conducted on datasets. To comprehend what are the items obtained by the users from online stores are analyzed effectively. The Scope of a project is to develop efficient techniques for user convenience, to handle the data products effectively, without setting the threshold value.

Objectives of Proposed system are 
$>$ It ought to be straightforward.

$>$ Easy to set up, easy to learn and utilize.

$>$ Making it simple to discover individuals and data.

$>$ Can sort out data by individuals, topics and so forth.

$>$ It should ready to utilize successfully by PC learners and specialists.

$>$ Online Collaboration System straightforward and capable.

$>$ It should make online cooperation speedier and less demanding.

$>$ Information ought to be secure.

\section{SYSTEM ARCHITECTURE}

\subsection{Architecture Diagram}

Figure 2 represents the basic system architecture functionality of the system. High utility datasets, main intension of the system is to reduce the datasets over calculated profits to construct the algorithms. The architecture design to mining the result with High utility pattern growth algorithm obtains from the databases. Whereas the general process is that user in the related webpage and for login register their data then gets the access to search for more products and the data information are stored in the database. For mining the results the algorithm of high utility are used for mining process.

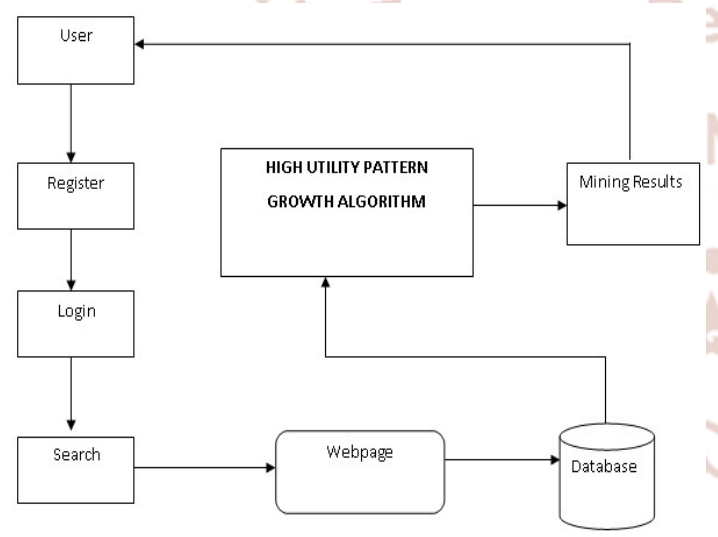

Figure 2 represents Basic Architecture Diagram For Proposed System Plan

\subsection{Flow Diagram}

Figure 3 represents the Flow diagram of the system with work flow activity and sequential representation of people actions or things that involves with conditions. For instance, considering user with conditions applied as "If new user" then the user wants to register their details in the respected fields and get their login. After that search of a product or items that are be stored in the system are referred and profit value (threshold) related products list values are given to compare with it, to get the desired result of profited value as outcome.

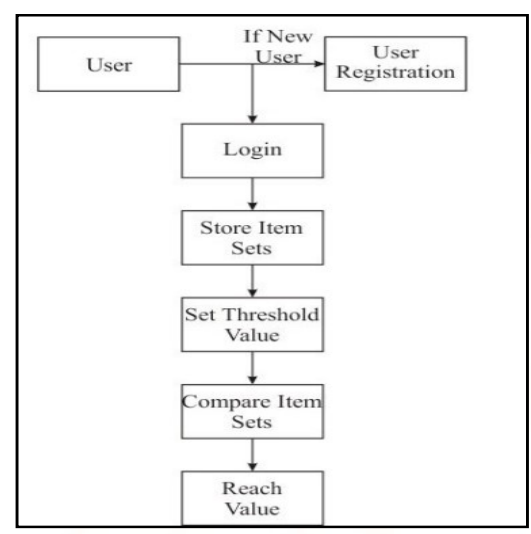

Figure 3 represents Flow Diagram

\subsection{Use Case Diagram}

Figure 4 represents the use case diagram of the system with user (actor) uses website to access it with login, product, high utility data, frequent items to buy and discount offers are used to make purchases.

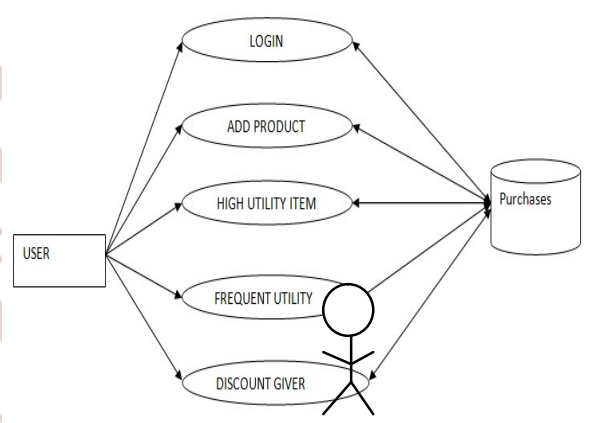

Figure 4 represents Use Case Diagram

\subsection{DATA FLOW DIAGRAMS (DFD)}

Figure 5 represents the Data Flow diagram of the system. It is a graphical instrument, which has the reason for clearing up framework prerequisites and distinguishing significant change that will be programs in the framework outline and also it provides an instrument for functional modeling and data stream demonstrating.

A DFD representation by an outer entity which can be a source or a predetermination is represented by a strong square. It lies outside the context of the framework. A procedure demonstrates the work that is performed on information. A circle represents a procedure. Information (Data flow) takes place between different segments of the framework and it is spoken to by an arrow mark. A data store is an archive for data. It is represented by an open finished rectangle. 


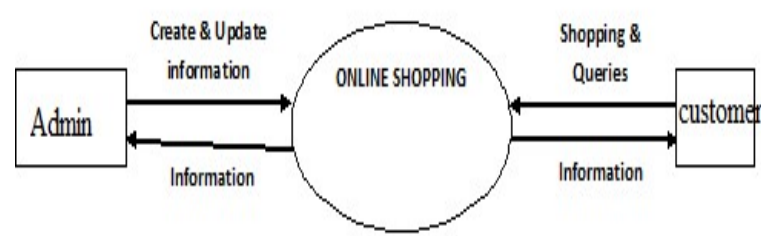

Figure 5 represents Level 0 DFD

\section{PROPOSED SYSTEM}

The basic idea of Top-k utility model was introduced to make the performance of the mining function and used for mining all high utility datasets. TKU gives a new technique in analyzing the datasets. The datasets with both high frequent and high utility mining can be obtained using utility methods. In existing affiliation rule mining used to distinguish much of the time happening designs thing set. ARM model treats every one of the data in the database equally by just considering, if a data is available in transaction or not. The frequent item set mining methodology may not fulfill sales chief's objective.

The Proposed system with the Customer Relationship Management is one of the methods in the system that fused into the system by tracking the customers who are frequent visitor purchasers of the different kinds of datasets and to improve the system performance by effective rating with POS tagging calculation and Opinion mining calculation to grip the related data. To reduce the computational time the authors present the lingering trees. The Datasets that are both high frequent and high utility can be gotten utilizing the strategy. Users are required to enlist on the site before they can do the shopping. The site likewise gives a few highlights to the non registered user. Here they can pick their id and every one of the insights with respect to them is gathered and a mail is sent to the email address or SMS to enlisted mobile number for affirmation. Thus the customer relationship management deals with the system by tracking details and information given to the customers. In this the admin find the frequent users data and gives discount for the product. Using this customer relationship will be maintained. User's frequent purchasing product can easily identified by the admin. Through this fast moving product details can be identified. For effective system performance the algorithms used are

Part-of-Speech (POS) Tagging Algorithm:

Fixing grammatical tags to words

$>$ Uncertainty: "tag" could be a naming verb or a word
$>$ "a tag is a part-of-speech marker" resolves the uncertainty

$>$ Word identification, substance extraction, etc.

\section{Opinion mining:}

Opinion mining is a type of normal dialect and it is also known as assessment analysis. . It is utilized for tracking the disposition of people in general about a specific item. Additionally includes building a framework to gather and arrange assessments about an item. Automated opinion mining frequently utilizes machine taking in, a kind of artificial brainpower, to mine the content for opinion.

\section{IMPLEMENTATION RESULTS AND DISCUSSION}

\subsection{User Registration Form}

The Figure 6 shows the user registration form according to the required fields. The fields include username, password, confirm password, first name, last name, e-mail, address, phone number. After registration the user will be directed to the main home page.

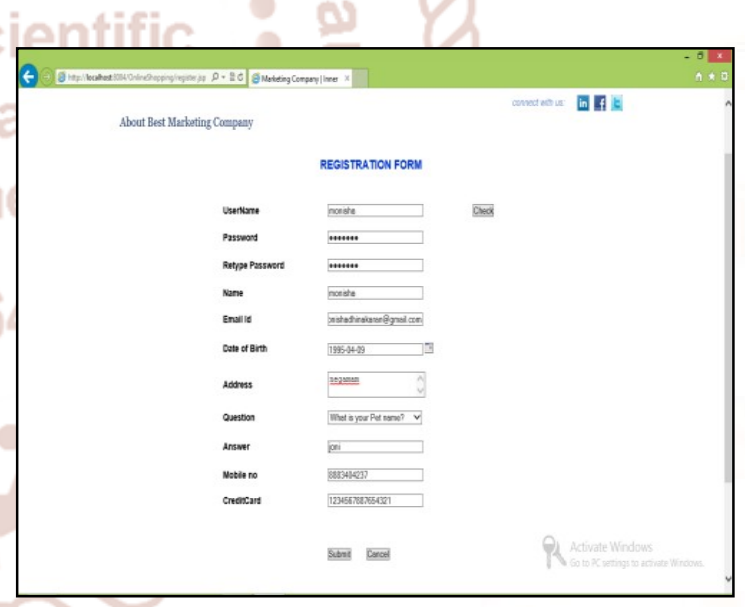

Figure 6 represents User Registration Form

\subsection{User Module}

The Figure 7 shows the user login page for new user account creation. In the login page, the user wants to get access to all the functionalities of online product Store. Login using user name and password. The user enters username and password, if it is a successful login the user will be directed to the menu page. Else if the user enters invalid information will be asked to check the entered information. 


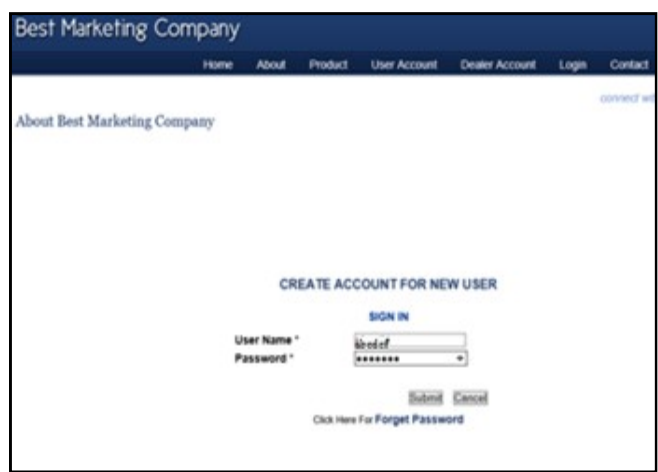

Figure 7 represents User Login

\subsection{Product Search}

The Figure 8 shows that search product of choice by selecting category and title. Then the selected product retrieves data from the database and displays the selected information. The system will display the products which matches the selected search criteria. A dataset is created as a result of select query. This search facility is given to both registered and unregistered user. User can scan for the accessibility and kind of items accessible on the site.

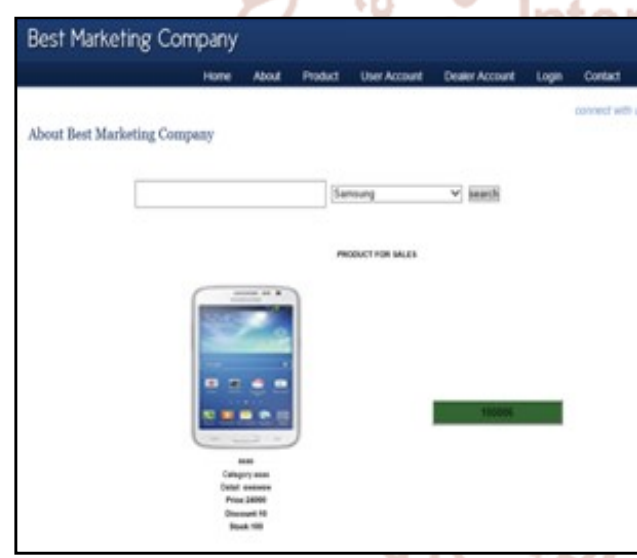

Figure 8 represents Search for a Product

\subsection{Setting Threshold Value (existing system)}

The Figure 9 shows setting of minimum utility threshold value to search a product list. If the threshold value is set to be too low, too many product items are displayed, which may result in the mining process very ineffectual. And also, if the threshold value is set to be too high, it results with no products be displayed.

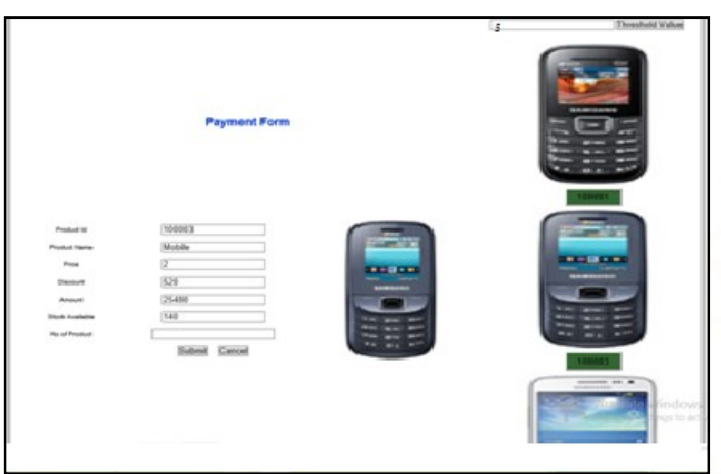

Figure 9 represents Setting Threshold Value

\subsection{Without setting threshold value (proposed system)}

The Figure 10 shows that search product of an item by selecting category and title without setting threshold value to the product. In which the proposed system displays with related product list which may result in the mining process very effectual. The Related products that are similar to chosen product with product price, product quality and other featured matched products are to be displayed over there.

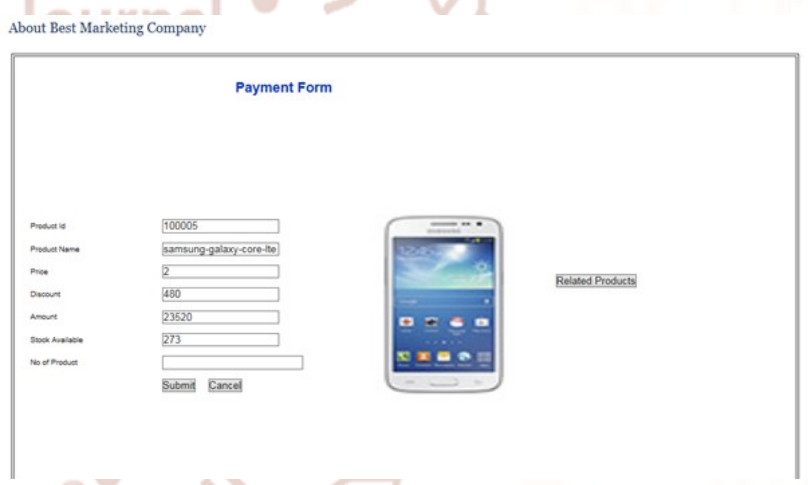

Figure 10 represents Related Product items to be displayed

\subsection{Give Rating to a Product}

The Figure 11 shows the rating of a product based on the customer's opinion and the customers can also rate the product or service through a feedback icon near the product. If the user wants to give rating according to his opinion for a product and can select either Good, Better, Best, and Worst. The final rating of a product will depend on all the individual user rating. The system will display the rating of a product and the total number of votes received. They can either rate or add description as feedback. 
Best Marketing Company

About Best Marketing Cocupanyox

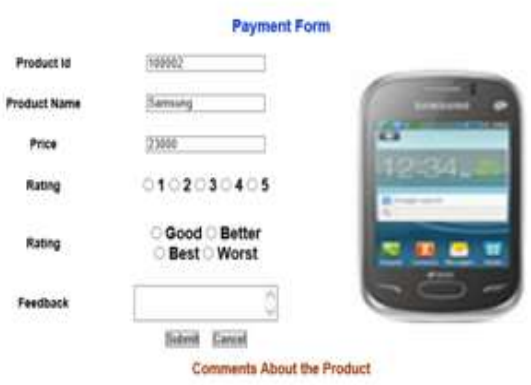

Figure 11 represents Rating to a Product

\subsection{Report details}

The Figure 12 shows the Users report of a product based on the product name, product type and product count details are to be reported.

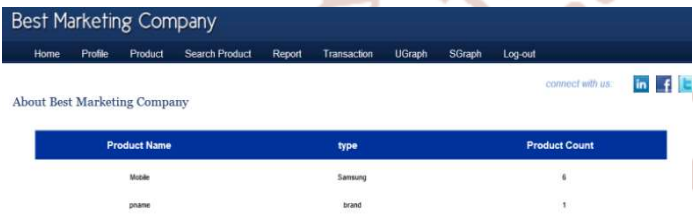

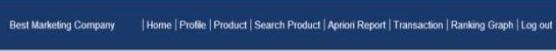

Figure 12 represents User Report

\subsection{Transaction details}

The Figure 13 shows the Users transaction details of a product, based on the product lists, price, Discount, amount to be paid are listed in the transaction table.

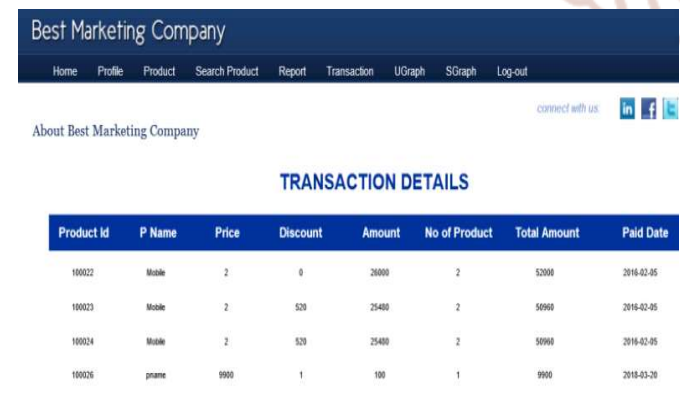

Figure 13 represents User Transaction details

\subsection{U - Graph (Utility)}

The Figure 14 shows the Utility Graph of a product whereas utility is the aggregate fulfillment got from all units of a specific product consumed over some stretch of time.

For instance, the customer devours mobiles and picks up 30 numbers of aggregate utility. This aggregate utility is the total of utilities from the progressive units (15 numbers from the primary mobiles, 10 numbers from the second and 5 numbers from the third brand mobiles). Adding up to utility is the measure of fulfillment (utility) acquired from expending a specific amount of a decent or administration inside a given day and period. It is the entirety of minimal utilities of each progressive unit of utilization.

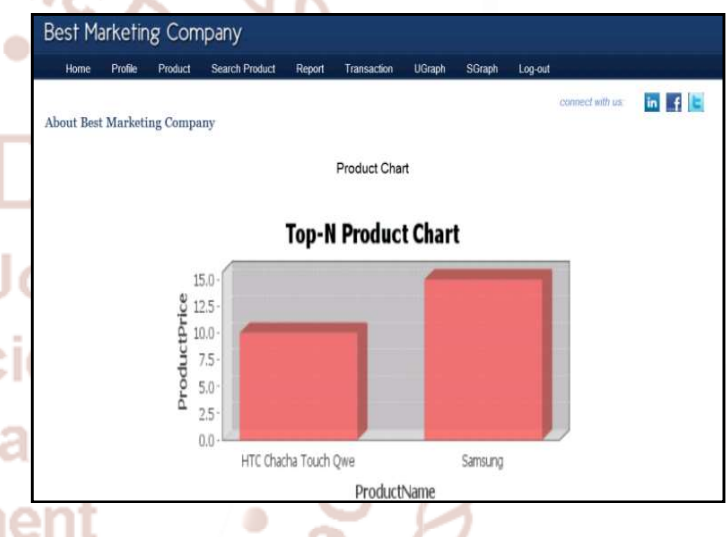

Figure 14 represents Utility Graph

\subsection{S - Graph (Sales)}

The Figure 15 shows that the level pivot on the chart demonstrates the quantity of units sold i.e. Samsung (product name). The vertical level demonstrates the quantity of units sold and is estimated in numbers which go up by product price ( 0 to 5 ) increment at each level. The chart appears to demonstrate that business figures have gone up and down finished the period portrayed.

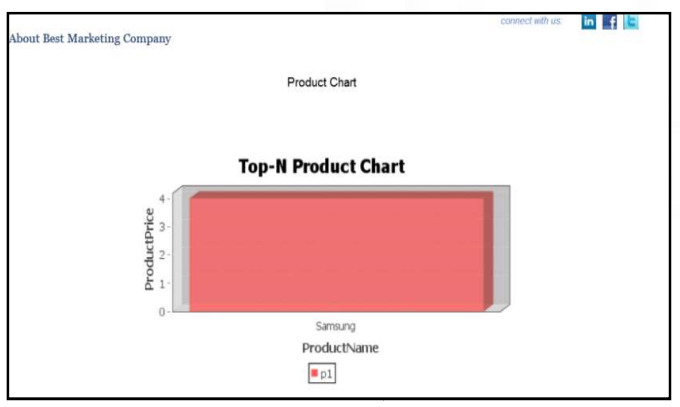

Figure 15 represents Sales Graph 
International Journal of Trend in Scientific Research and Development (IJTSRD) ISSN: 2456-6470

\section{CONCLUSION}

In data mining, Utility Mining relates utility considerations during dataset mining. The problems by proposing a new idea for top-k high utility dataset mining, where $\mathrm{k}$ is the desired number of HUDs to be mined. The operative calculation mining with High Utility datasets used for mining those datasets without the need of setting minimum utility. The datasets are obtained by calculating the absolute utilities of HUDs with one database view. These are used for mining the complete set of HUDs in databases without the need to specify the lower profit threshold. Evaluate estimate on both certified and simulated datasets shows the activity of the advanced algorithms around the most effective case in utility mining algorithms.

The present system discusses with user, administrator and dealer methodologies. The customer process includes account creation, add or delete of a product, the customer details are stored in the database to get customer transaction graph and profit graph. The user process includes registration form, search of a product by setting threshold value as existing system and no need of setting threshold value be proposed with related product items are displayed then giving feedbacks comments to the products and rating to a product are executed with the Rystem implementations. The proposed system, it includes with the Customer Relationship Management will be incorporated into the system by tracking the customers who are frequent buyers of the different kinds of datasets and to improve the system performance by effective rating with POS tagging calculation and Opinion Mining calculation on advanced efficient techniques to grip the related data.

\section{REFERENCES}

1. Yamini.P,Soma Shekar,J.Deepthi,(2017) "Efficient Algorithms For Mining Top-K High Utility Itemset" International Journal Of
Computational Science, Mathematics And Engineering Volume-4-Issue- Issn-2349-8439.

2. Vincent S. Tseng, Cheng-Wei Wu, Philippe Fournier-Viger, and Philip S. Yu, Fellow,(2016) "Efficient Algorithms For Mining Top-K High Utility Itemset". IEEE Transactions on Knowledge and Data Engineering, Vol. 28, No. 1.

3. Komal Surawase, Madhav Ingle,(2015) "High Utility Itemset Mining From Transaction Database Using Up-Growth And Up-Growth+ Algorithm", International Journal Of Innovative Research In Computer And Communication Engineering (An ISO 3297:2007 Certified Organization) Vol.3, Issue 4.

4. Shipra Khare, Prof.Vivek Jain,(2014) "A Review On-Infrequent Weighted Itemset Mining Using Frequent Pattern Growth" (IJCSIT) International Journal Of Computer Science And Information Technologies, Vol. 5 (2).

5. U Kanimozhi. J.K. Kavitha, D.Manjula,(2014) "Mining High Utility Itemset-A Recent Survey" Journal of Scientific Engineering and Technology (ISSN: 2277-1581) Volume No.3 Issue No.11, 01 Nov.

6. Vincent S.Tseng, Bai-En Shie, Cheng-Wei Wu, and Philip S. Yu, Fellow,(2013) "Efficient Algorithms for Mining High Utility Itemset from Transactional Databases" IEEE Transactions on Knowledge and Data Engineering, Vol. 25, No.8.

7. Cheng Wei Wu, Bai-En Shie, Philip S. Yu, Vincent S. Tseng "Mining Top-K High Utility Itemset", National Cheng Kung University, Taiwan, Roc.

8. Nilovena.K.V, Anu.K.S (2012), "Study on High Utility Itemset Mining", (IJCSIT) International Journal of Science And Research.

9. Archana Kisan Dere (2016), "Survey On Techniques Of High Utility Mining". 